\title{
Correspondence
}

Editor: Ian Pullen

Contents: CPK in NMS/Understanding Capgras syndrome/Eating disorder in Asian women/Transsexualism/Carbamazepine in alcohol withdrawal/ Culture as a confounding variable?/Buspirone in detoxification/Is psychiatric training still improving?/Are British psychiatrists racist?/ECT following clozapine/Buspirone-induced mania/Educating the psychiatrist of the 21 st century

\section{CPK in NMS}

SIR: We are concerned at the inappropriate emphasis being placed on creatinine phosphokinase (CPK) estimation in the diagnosis of neuroleptic malignant syndrome (NMS) and in its differential diagnosis from other disorders. We concur with Gratz \& Simpson (Journal, October 1990, 157, 617-618) that elevated levels of CPK may occur as a result of untreated rigidity and wish to emphasise that this need not necessarily be induced by neuroleptics. The recent case report by Dalkin \& Lee (Journal, September 1990, 157, 437-438) states that their diagnosis of NMS "rests" on the raised CPK, and they dismiss the possibility that this may be the result of severe dystonia because "there are no reports in the literature of neuroleptic-induced dystonic reactions causing raised CPK levels". Levenson (1985) includes elevated CPK as one of three major criteria of diagnostic importance in NMS and implies that raised CPK differentiates NMS from catatonia. Some other authors have, however, been more cautious about using CPK measurements in such a definitive fashion. Adityanjee et al (1988) stress that, when diagnosing NMS, CPK and leucocytosis should be used only in support of the clinical findings.

We report a case of catatonia in which CPK estimates reflected the clinical picture.

Case report: A 19-year-old single woman with no past psychiatric or medical history of note developed an acute paranoid psychosis with progressive motor disturbances including stereotyped rocking, facial grimacing and withdrawal, followed by stupor with incontinence. Four days after the onset she became rigid, postured for long periods and displayed waxy flexibility and resistance. This was interrupted by short periods of excited motor behaviour and high pitched squeals. On admission all blood results including full blood count, erythrocyte sedimentation rate and CPK were normal, as was a urine drug screen. As the catatonic movements became more severe, her CPK rose reaching a maximum of $1220 \mathrm{iu} / \mathrm{l}$ six days after admission. Twelve days after admission electroconvulsive therapy was commenced because of her continued catatonic state with no oral food or fluid intake. Her condition responded rapidly and after seven treatments she was completely recovered. At one week following recovery, CPK had returned to within normal limits $(0-60 \mathrm{iu} / \mathrm{l})$. At no time before or during this patient's illness did she receive neuroleptic or other psychotropic medication.

CPK is found in muscle and brain tissue and provides a measure of tissue catabolism. There are three isoenzymes, of which CPK MM most closely reflects muscle activity and integrity. CPK MM may be raised by skeletal muscle damage (including intramuscular injections, exercise, trauma and massage) and by some drugs including succinylcholine, alcohol and lithium. It is also raised in malignant hyperpyrexia, hypothermia, coma, infectious diseases involving muscle, convulsions, hypothyroidism and muscular rigidity. It may be raised in extreme muscular activity due to agitation as seen in psychotic patients (Ford, 1987).

Our case is a clear demonstration that CPK may be elevated when catatonic symptoms occur in the absence of NMS. We believe that, when diagnosing NMS, it is inappropriate to put undue emphasis on the presence of elevated CPK which is, after all, simply a non-specific indicator of muscular catabolism.

BRIDGet CRADDOCK

Nick CRADDOCK

GeORGE MiLNER

Worcester Royal Infirmary

Newtown Road

Worcester WR5 1 JG

\section{References}

Adityanjee, Singh S., Singh, G., et al (1988) Spectrum concept of neuroleptic malignant syndrome. British Journal of Psychiatry, 153, 107-111.

FORD, R. D. (1987) Diagnostic Tests Handbook. Springhouse, Pennsylvania: Springhouse Corporation.

LEVENSON, J. L. (1985) Neuroleptic malignant syndrome. American Journal of Psychiatry, 142, 1137-1145. 